\title{
Complex Method on Octagonal Number
}

\section{S. Sudha, A. Gnanam}

Abstract: In Number theory Study of polygonal numbers is rich in varity. In this paper we establish a Complex Octagonal Number using Continued Fraction algorithm.

Keywords: Algorithm, Continuedfraction, complex Number. Octagonal Number.

\section{INTRODUCTION}

A Simple continued fraction [1] is an expression of the form

$$
a_{0}+\frac{b_{0}}{a_{1}+\frac{b_{1}}{a_{2}+\frac{b_{2}}{\ddots}}}
$$

Where the $a_{i}$ are a possibly infinite sequence of integers such that $a_{1}$ is non-negative and the rest of the sequence is positive. We write $\left\langle a_{1} ; a_{2}, a_{3} \ldots \ldots\right\rangle$. The above fraction also calls them Regular continued fractions.

\section{CONTINUED FRACTION ALGORITHM}

Suppose we wish to find continued fraction expansion[2] of $x \in R$.

Let $x_{0} \in x$ and set $a_{0}=\left[x_{0}\right]$, $a_{2}=\left[x_{2}\right] \ldots x_{k}=\frac{1}{x_{k-1}-\left[x_{k-1}\right]} \Rightarrow a_{k}=\left[x_{k}\right] \ldots$

This process is continued infinitely or to some finite stage till an $x_{i} \in N$ exists such that $a_{i}=\left[x_{i}\right]$.

\section{OCTAGONAL NUMBER}

A. Definition: Centered Octagonal Number[3] The Number $1,9,25,49,81,121, \ldots \ldots \ldots$ are called centered octagonal numbers. The number that represents associate in nursing polygonal shape with a dot within the center and every one dots different dots encompassing the middle dot in associate in nursing polygonal shape lattice . formula

The $n^{\text {th }}$ centered octagonal number is given by the $O_{n}=4 n(n-1)+1$
Define $x_{1}=\frac{1}{x_{0}-\left[x_{0}\right]}$ and set $a_{1}=\left[x_{1}\right]$ and $x_{2}=\frac{1}{x_{1}-\left[x_{1}\right]} \Rightarrow$

\section{B. Theorem:}

For $n \geq 3$, $\frac{O_{n}}{O_{n+1}}+i \frac{O_{n+2}}{O_{n+3}}$

$=\left\{\begin{array}{c}\left\langle 0 ; 1,\left[\frac{n}{2}\right], 8 n\right\rangle+i\left\langle 0 ; 1,\left[\frac{n+1}{2}\right], 8(n+2)\right\rangle \text { when } n \text { is odd } \\ \left\langle 0 ; 1, \frac{n}{2}-1,1,1,2 n-1,2\right\rangle+i\left\langle 0 ; 1, \frac{(n+2)}{2}-1,1,1,2(n+2)-1,2\right\rangle \text { when } n \text { is even }\end{array}\right.$

Proof:

Case (i): $-\mathrm{n}$ is odd

Let $\mathrm{n}=2 \mathrm{k}-1$, Where $3 \leq k \leq n$

Then

$\frac{O_{2 k-1}}{O_{2 k}}+i \frac{O_{2 k+1}}{O_{2 k+2}}=\left\langle 0 ; 1,\left[\frac{2 k-1}{2}\right], 8(2 k-1)\right\rangle$ $+i\langle 0 ; 1,[k], 8(2 k+1)\rangle$

Next we have to prove that $\mathrm{n}=2 \mathrm{k}+1$

To find the continued fraction of

$\frac{O_{2 k+1}}{O_{2 k+2}}+i \frac{O_{2 k+3}}{O_{2 k+4}}$

\section{A. Real Part:-[3]}

$$
\begin{aligned}
\frac{O_{2 k+1}}{O_{2 k+2}} & =\frac{4(2 k+1)(2 k+1-1)+1}{4(2 k+2)(2 k+2-1)+1} \\
& =\frac{16 k^{2}+8 k+1}{16 k^{2}+2 k+9} \\
x_{0} & =\frac{16 k^{2}+8 k+1}{16 k^{2}+2 k+9}, a_{0}=0 \\
x_{1} & \Rightarrow 1+\frac{16 k+8}{16 k^{2}+8 k+1} \Rightarrow a_{1}=1 \\
x_{2} & \Rightarrow k+\frac{1}{16 k+8} \Rightarrow a_{2}=k \\
x_{3} & \Rightarrow 16 k+8 \Rightarrow a_{3}=16 k+8 \\
= & =8(2 \mathrm{k}+1) \\
\therefore & \frac{O_{2 k+1}}{O_{2 k+2}}=\langle 0 ; 1, k, 8(2 k+1)\rangle
\end{aligned}
$$

\section{B. Imaginary part:-}

$$
\begin{aligned}
& \frac{O_{2 k+3}}{O_{2 k+4}} \Rightarrow \frac{4(2 k+3)(2 k+3-1)+1}{4(2 k+4)(2 k+4-1)+1} \\
&=\frac{4\left[4 k^{2}+6 k-2 k+6 k+9-3\right]+1}{4\left[4 k^{2}+8 k-2 k+8 k+16-4\right]+1} \\
&=\frac{4\left[4 k^{2}+4 k+6 k+6\right]+1}{4\left[4 k^{2}+14 k+12\right]+1} \\
&=\frac{4\left[4 k^{2}+10 k+6\right]+1}{4\left[4 k^{2}+14 k+12\right]+1} \\
&=\frac{16 k^{2}+40 k+24+1}{16 k^{2}+56 k+48+1} \\
& \frac{O_{2 k+3}}{O_{2 k+4}} \Rightarrow \frac{16 k^{2}+40 k+25}{16 k^{2}+56 k+49} \\
& x_{0}=\frac{16 k^{2}+40 k+25}{16 k^{2}+56 k+49} ; a_{0}=0
\end{aligned}
$$

Then

$$
x_{1} \Rightarrow \frac{16 k^{2}+56 k+49}{16 k^{2}+40 k+25}=1+\frac{16 k+24}{16 k^{2}+40 k+25} \Rightarrow a_{1}=1
$$

\footnotetext{
S. Sudha, Department of Mathematics, Nazareth College of Arts and Science, Chennai - 62, Research Scholar, Department of Mathematics, Government Arts College, Trichy- 22.
}

A. Gnanam, Department of Mathematics, Government Arts College, Trichy- 22. 


\section{Complex Method on Octagonal Number}

$$
\begin{gathered}
x_{2} \Rightarrow \frac{16 k^{2}+40 k+25}{16 k+24}=(k+1)+\frac{1}{16 k+24} \Rightarrow a_{2} \\
x_{3} \Rightarrow \frac{16 k+24}{1} \Rightarrow 16 k+24 \\
\Rightarrow 8(2 k+3) \\
\therefore \frac{O_{2 k+3}}{O_{2 k+4}}=\langle 0 ; 1,(k+1), 8(2 k+3)\rangle \\
\therefore \frac{O_{2 k+1}}{O_{2 k+2}}+i \frac{O_{2 k+3}}{O_{2 k+4}} \\
=\langle 0 ; 1, k, 8(2 k+1)\rangle \\
+i\langle 0 ; 1,(k+1), 8(2 k \\
+3)\rangle
\end{gathered}
$$

By the results is true for all values of $n$ when $n$ is odd.

$$
\text { Case (ii): } n \text { is even }
$$

Let $\mathrm{n}=2 \mathrm{k}-2$

Then

$$
\begin{aligned}
\frac{O_{2 k-2}}{O_{2 k-1}}+i \frac{O_{2 k}}{O_{2 k+2}}= & \left\langle 0 ; 1, \frac{2 k-2}{2}-1,1,1,2(2 k-2)\right. \\
& -1,2\rangle \\
& +i\left\langle 0 ; 1, \frac{2 k-2+2}{2}-1,1,1,2(2 k-2)\right. \\
& -1,2\rangle \\
& =\langle 0 ; 1, k-2,1,1,2(2 k-2)-
\end{aligned}
$$

\section{$1,2+i 0 ; 1, k-1,1,1,22 k-2-1,2$}

Next we have to prove that $\mathrm{n}=2 \mathrm{k}$

To find the continued fraction of

$$
\frac{O_{2 k}}{O_{2 k+1}}+i \frac{O_{2 k+2}}{O_{2 k+3}}
$$

\section{Real Part:-[3]}

$\frac{O_{2 k}}{O_{2 k+1}}=\langle 0 ; 1, k-1,1,1,4 k-1,2\rangle$

\section{Imaginary part:-}

$$
\begin{aligned}
& \frac{O_{2 k+2}}{O_{2 k+3}}= \frac{4(2 k+2)(2 k+2-1)+1}{4(2 k+3)(2 k+3-1)+1} \\
&=\frac{4\left[4 k^{2}+2 k+4 k+2\right]+1}{4\left[4 k^{2}+4 k+6 k+6\right]+1} \\
&=\frac{4\left[4 k^{2}+6 k+2\right]+1}{4\left[4 k^{2}+10 k+6\right]+1} \\
&=\frac{16 k^{2}+24 k+8+1}{16 k^{2}+40 k+24+1} \\
&=\frac{16 k^{2}+24 k+9}{16 k^{2}+40 k+25} \\
& \frac{O_{2 k+2}}{O_{2 k+3}} \Rightarrow \frac{16 k^{2}+24 k+9}{16 k^{2}+40 k+25} \\
& x_{0}=\frac{16 k^{2}+24 k+9}{16 k^{2}+40 k+25} ; a_{0}=0
\end{aligned}
$$

Then

$$
\begin{aligned}
x_{1} & \Rightarrow \frac{16 k^{2}+40 k+25}{16 k^{2}+24 k+9}=1+\frac{16 k+16}{16 k^{2}+24 k+9} \Rightarrow a_{1}=1 \\
x_{2} & \Rightarrow \frac{16 k^{2}+24 k+9}{16 k+16}=k+\frac{8 k+9}{16 k+16} \Rightarrow a_{2}=k \\
x_{3} & \Rightarrow \frac{16 k+16}{8 k+9} \Rightarrow 1+\frac{8 k+7}{8 k+9} \Rightarrow a_{3}=1 \\
x_{4} & \Rightarrow \frac{8 k+9}{8 k+7} \Rightarrow 1+\frac{2}{8 k+7} \Rightarrow a_{4}=1 \\
x_{5} & \Rightarrow \frac{8 k+7}{2} \Rightarrow(4 k+3)+\frac{1}{2} \Rightarrow a_{5}=4 k+3 \\
x_{6} & \Rightarrow \frac{2}{1} \Rightarrow a_{6}=2 \\
\therefore & \left.\frac{O_{2 k+2}}{O_{2 k+3}}=\langle 0 ; 1, k, 1,1,4 k+3,2)\right\rangle
\end{aligned}
$$

Since

$\frac{O_{2 k}}{O_{2 k+1}}+i \frac{O_{2 k+2}}{O_{2 k+3}}=\langle 0 ; 1, k-1,1,1,4 k-1,2\rangle$ $+i\langle 0 ; 1, k, 1,1,4 k+3,2)\rangle$

Hence by the result is true for all value of $n$ where $n$ is even.

Since case (i) \& case (ii) for each $n \geq 3$, the continued fraction expansion of

$$
\begin{aligned}
& \frac{O_{n}}{O_{n+1}}+i \frac{O_{n+2}}{O_{n+3}} \\
& =\left\{\begin{array}{l}
\left\langle 0 ; 1,\left[\frac{n}{2}\right], 8 n\right\rangle+i\left\langle 0 ; 1,\left[\frac{n+1}{2}\right], 8(n+2)\right\rangle \text { when } n \text { is odd } \\
\left\langle 0 ; 1, \frac{n}{2}-1,1,1,2 n-1,2\right\rangle+i\left\langle 0 ; 1, \frac{(n+2)}{2}-1,1,1,2(n+2)-1,2\right\rangle \text { when } n \text { is even }
\end{array}\right.
\end{aligned}
$$

\section{ILLUSTRATION}

Let $n=3$,

$\frac{O_{3}}{O_{4}}+i \frac{O_{5}}{O_{6}}=\frac{25}{49}+i \frac{81}{121}$

A. Real Part:-[3]

$\frac{O_{3}}{o_{4}}=\frac{25}{49}$, so $a_{0}=0$

$\therefore x_{0}=\frac{25}{49}$

Then $x_{1}=\frac{1}{x_{0}-\left[x_{0}\right]}=\frac{49}{25}=1+\frac{24}{25} \Rightarrow a_{1}=1$

$$
\begin{gathered}
x_{2}=\frac{1}{x_{1}-\left[x_{1}\right]}=\frac{25}{24}=1+\frac{1}{24} \Rightarrow a_{2}=1 \\
x_{3}=\frac{1}{x_{2}-\left[x_{2}\right]}=\frac{24}{1}=24 \Rightarrow a_{3}=24 \\
\therefore \frac{25}{49}=\langle 0 ; 1,1,24\rangle
\end{gathered}
$$

B. Imaginary Part:-

$\frac{O_{5}}{o_{6}}=\frac{81}{121}, a_{0}=0$
$x_{0}=\frac{81}{121}, a_{0}=0$

Then

$x_{1}=\frac{1}{x_{0}-\left[x_{0}\right]}=\frac{121}{81}=1+\frac{40}{81} \Rightarrow a_{1}=1$

$x_{2}=\frac{1}{x_{1}-\left[x_{1}\right]}=\frac{81}{40}=2+\frac{1}{40} \Rightarrow a_{2}=2$

$x_{3}=\frac{1}{x_{2}-\left[x_{2}\right]}=\frac{40}{1}=40 \Rightarrow a_{3}=40$

$\frac{81}{121}=\langle 0 ; 1,2,40\rangle$

Hence

$\frac{25}{49}+i \frac{81}{121}=\langle 0 ; 1,1,24\rangle+i\langle 0 ; 1,2,40\rangle$

\section{ILLUSTRATION}

Put $\mathrm{n}=4$

$\frac{O_{4}}{O_{5}}+i \frac{O_{6}}{O_{7}}=\frac{49}{81}+i \frac{121}{169}$

A. Real Part:-[3]

$\frac{O_{4}}{O_{5}}=\frac{49}{81} \Rightarrow\langle 0 ; 1,1,1,1,7,2\rangle$

B. Imaginary part:-

$\frac{O_{6}}{O_{7}}=\frac{121}{169}$

Let $x_{0}=\frac{121}{169}, a_{0}=0$

Then 


$$
\begin{aligned}
& x_{1}=\frac{1}{x_{0}-\left[x_{0}\right]}=\frac{169}{121}=1+\frac{48}{121} \Rightarrow a_{1}=1 \\
& x_{2}=\frac{1}{x_{1}-\left[x_{1}\right]}=\frac{121}{48}=2+\frac{25}{48} \Rightarrow a_{2}=2 \\
& x_{3}=\frac{1}{x_{2}-\left[x_{2}\right]}=\frac{48}{25}=1+\frac{23}{25} \Rightarrow a_{3}=1 \\
& x_{4}=\frac{1}{x_{3}-\left[x_{3}\right]}=\frac{25}{23}=1+\frac{2}{23} \Rightarrow a_{4}=1 \\
& x_{5}=\frac{1}{x_{4}-\left[x_{4}\right]}=\frac{23}{2}=11+\frac{1}{2} \Rightarrow a_{5}=11 \\
& x_{6}=\frac{1}{x_{5}-\left[x_{5}\right]}=2 \Rightarrow a_{6}=2 \\
& \frac{121}{169}=\langle 0 ; 1,2,1,1,11,2\rangle
\end{aligned}
$$

Since

$$
\begin{aligned}
\frac{49}{81}+i \frac{121}{169} & \\
& =\langle 0 ; 1,1,1,1,7,2\rangle \\
& +i\langle 0 ; 1,2,1,1,11,2\rangle
\end{aligned}
$$

\section{CONCLUSION}

In this paper we have identified complex octagonal number using continued fractions.

\section{REFERENCES}

1. https://crypto.stanford.edu

2. International Journal of Engineering Science, Advanced Computing and Bio - Technology "Ratios of Polygonal Numbers as Continued Fractions" - A. Gnanam and S. Krithika.

3. The International Journal of analytical and experimental modal analysis " Algebraic Approach on Octagonal Numbers"- A.Gnanam and S. Sudha

\section{AUTHORS PROFILE}

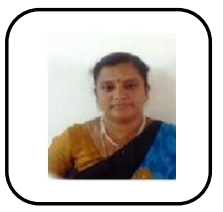

S. Sudha She received M.Sc. (Maths) Degree in Seethalakshmi Ramasamy College, Trichy in 2001, M.Phil (Maths) from ST. Joseph's College, Trichy in 2007, presently she is pursuing Ph.D. from Bharathidasan University, Trichy and working as Assistant Professor at Nazareth College of Arts \& Science, Avadi since December 2007. She started her career as Assistant Professor at Valluvar College of Science \& Management, Karur from March 2007 to Oct 2007. She has teaching experience of 13 years. She has handled Allied Mathematics, Algebraic Structures, Numerical Analysis, Operations Research, Linear Algebra, Transform Technique and Analytical Geometry. She has participated in 19 and presented 7 papers in various National and International level Conference and Seminars. She has published 2 books with ISBN and one paper in International journal.

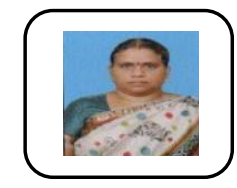

A. Gnanam was born in Trichy, Tamil Nadu, India in 1967. She received M.Sc., and M.Phil., degree in Annamalai University, Chithambaram. She got Ph.D degree in Mathematics from Bharathidasan University, Trichy. She started her career as a lecturer in 1993. Now she is working as an Assistant Professor in Mathematics in Government Arts College, Thuvakudi, Trichy. Her area of specialization is Number Theory. Dr. Gnanam published 25 papers in National and International journals. She produced two Ph.D, scholar and five more students are doing research under her guidance. 